\title{
Reconstrucción de los regímenes de fuego en un bosque de Prosopis caldenia, provincia de La Pampa, Argentina
}

\author{
Fire regime reconstruction in a Prosopis caldenia woodland, La Pampa, Argentina
}

\author{
Andrea A Medina \\ Universidad Nacional de La Pampa, Facultad de Ciencias Exactas y Naturales y Facultad de Agronomía, \\ Ruta 35, 6.300 Santa Rosa, La Pampa, Argentina, andrepampa@ hotmail.com
}

\begin{abstract}
SUMMARY
Since the introduction of forestry and livestock actitvities Prosopis caldenia (caldén) woodlands have undergone drastic structural changes. Fire has played an important role in such changes although cause-effect relationships have not been established conclusively. In this paper the fire regime that ocurred in a caldén woodland in estancia Bajo Verde, La Pampa Province, Argentina, is reconstructed, through dendrochronological analysis of fire scars recorded in sections of the trunk. The fire regime in the study area has been very dynamic during the $19^{\text {th }}$ and $20^{\text {th }}$ centuries. The important changes in frequency and areal extent that fires have experienced are probably related to changes in human occupation and land use patterns. This information would be useful for assessing the degree of influence of fire in the spatio-temporal variations in caldén woodlands.
\end{abstract}

Key words: fire frequency, fire scars, dendrochronology, disturbance.

\section{RESUMEN}

Los bosques de Prosopis caldenia (caldén) han sufrido grandes cambios estructurales a partir de la introducción de las actividades silvopastoriles en los mismos. El fuego ha jugado un rol importante en tales cambios aunque las relaciones de causa-efecto no han sido aún demostradas en forma conclusiva. En este trabajo se reconstruyen los regímenes de fuego ocurridos en un bosque de caldén de la estancia Bajo Verde, en la provincia de La Pampa, Argentina, mediante el análisis dendrocronológico de cicatrices de fuego registradas en el leño de los mismos. El régimen de fuego en el área de estudio ha sido muy dinámico durante los siglos XIX y XX. Tanto la frecuencia como la extensión de los fuegos han experimentado importantes cambios probablemente en relación a cambios en el tipo de ocupación y de uso del área. Esta información será de gran importancia a la hora de evaluar el grado de influencia del fuego en la variación espacio-temporal de los patrones de los bosques de caldén.

Palabras clave: Prosopis caldenia, frecuencia de fuego, cicatrices de fuego, dendrocronología, disturbios.

\section{INTRODUCCIÓN}

El fuego es reconocido como un importante componente del régimen de disturbios que afecta a los bosques de Prosopis caldenia Burkart (caldén) (Cano et al. 1985, Boó 1990, Gorondi 1990, Sipowicz 1994). Es muy común en estos bosques la existencia de caldenes, tanto muertos como vivos, que presentan marcas de carbonización en la corteza o troncos con grietas o cavidades originadas por sucesivas cicatrices de fuegos pasados. Las propiedades químicas y el grosor de la corteza del caldén contribuyen a su resistencia a la ignición (Willard y McKell 1977, Boó et al. 1997). Posee además la capacidad para brotar prolíficamente después de haber sido afectado por este disturbio y de compartimentalizar rápidamente las heridas, características que le confieren una ventaja competitiva en un ambiente con fuego (Brown y Swetnam 1993).

El distrito fitogeográfico del Caldenal (Cabrera y Willink 1973), que cubre en la actualidad unos 2,5 millones de ha en el centro de la Argentina, habría estado representado originalmente por un bosque abierto de caldén (Head 1918, De la Cruz 1969, Monticelli 1983) con árboles grandes dispersos en una matriz de pastizales de valor forrajero elevado (Llorens 2000). A partir de la introducción de las actividades silvopastoriles en la región, a fines del siglo XIX, este paisaje fue progresivamente invadido por formaciones arbustivas de la misma especie, denominadas localmente con el nombre de fachinales. Lell (1990), Scarone (1994), Steibel y Troiani (1999) y Llorens (2000) atribuyen parcialmente este fenómeno a los incendios y quemas sistemáticas que habrían acompañado a la colonización de la región. Por otro lado, Cano et al. (1985), Anchorena (1998), Boó (1990), Gorondi (1990), Sipowicz (1994) y Buzzo (1997) sostienen que la ausencia de fuego en el caldenal, o una reducción en su frecuencia a partir de la colonización europea, ha incentivado la "fachinalización" de estos bosques. Boó et al. (1997) sostienen que, debido a que el banco de semillas de esta leñosa es bajo, y la germinación y su establecimiento es 
infrecuente, el alto potencial de rebrote del caldén después del fuego podría contribuir a su persistencia en la comunidad. Los mismos autores sostienen que la comunidad del caldenal sería resiliente al fuego.

Este debate es de importancia para el manejo de las comunidades de caldén, particularmente en lo que concierne a la planificación en el uso del fuego y su manejo, dada su gran valoración socioeconómica y ecológica en la región. De importancia central en esta discusión es la naturaleza histórica del fuego en los bosques de caldén.

El régimen de fuegos es una combinación de componentes temporales y espaciales que se describe según sus características inmediatas, como la frecuencia, la intensidad, la extensión y la estacionalidad. La frecuencia de fuegos resulta una variable especialmente importante a considerar en la dinámica de los bosques ya que ésta determina en gran parte su composición y su estructura (Lorimer 1985, Brown y Sieg 1996, Lehtonen et al. 1996, Lara et al. 1999, 2003). Aun así, la estacionalidad, la extensión y la intensidad de los fuegos forestales tienen también una influencia decisiva en el curso de las sucesiones (Whelan 1995).

El análisis dendrocronológico de cicatrices de fuego permite la reconstrucción histórica y la descripción de las características inmediatas del régimen de fuegos que han afectado a ecosistemas boscosos (McBride 1983, Lara et al. 1999, Kitzberger et al. 2000, González et al. 2005) y específicamente a los bosques de caldén (Medina et al. 2000, Medina 2003).

Este trabajo tiene como objetivo reconstruir los regímenes de fuego y caracterizar algunas de sus variables, principalmente la frecuencia y la estacionalidad, ocurridos en un bosque de caldén de la estancia Bajo Verde, departamento de Toay, provincia de La Pampa, Argentina, mediante el análisis dendrocronológico de cicatrices de fuego. Esta información será de importancia a la hora de evaluar el grado de influencia del fuego y sus variables en la dinámica espacio-temporal de los bosques de caldén.

\section{MÉTODOS}

Área de estudio. Las muestras para este estudio fueron recolectadas a partir de árboles volteados durante la construcción de picadas cortafuegos en un área de 200 hectáreas de bosque de caldén de la estancia Bajo Verde (36 $27^{\prime}$ S, 6439' O), departamento de Toay, provincia de La Pampa, Argentina. Estos bosques pertenecen al distrito fitogeográfico del Caldenal de la Provincia del Espinal (Cabrera y Willink 1973). El área de estudio está representada por un bosque secundario con relictos de pequeña extensión del bosque prístino. Durante las últimas décadas la única actividad desarrollada en el área ha sido la cría de ganado vacuno. El área se encuentra a la altitud de 200 m s.n.m. y el régimen de precipitaciones es monzónico, con una media anual de $500 \mathrm{~mm}$, produciéndose los mayores valores de déficit hídrico en los meses de verano (diciembre a marzo). La temperatura media anual es de $15,5{ }^{\circ} \mathrm{C}$, mientras que para los meses más fríos (junio a septiembre) es de $8{ }^{\circ} \mathrm{C}$ y de $23,2{ }^{\circ} \mathrm{C}$ para los meses de verano (Cano et al. 1985).

Recolección y procesamiento de muestras. Para el muestreo fueron seleccionados los caldenes de mayor porte y con múltiples cicatrices de fuego, evidenciadas en los troncos por rebordes paralelos y sucesivos del leño. Esta estrategia de colección fue utilizada con el fin de maximizar el registro de fuegos de cada ejemplar muestreado y extender temporalmente la cronología de los mismos (Kitzberger et al. 2000).

A los árboles elegidos se les extrajo una sección transversal basal, cada una de las cuales fue pulida con lijas de granulometría ascendente. El fechado de los anillos de crecimiento se realizó bajo lupa estereoscópica sobre dos a tres radios distintos. De acuerdo a lo convenido entre los dendrocronólogos del hemisferio sur, el año calendario de cada anillo de crecimiento fue asignado al año en el cual el anillo comenzó a formarse (Schulman 1956). Se midieron las series de ancho de anillos anuales con equipo de precisión, se realizaron los controles de la calidad de las medidas mediante el uso del programa COFECHA (Holmes 1983) y luego se fecharon las cicatrices de fuego (Dieterich y Swetnam 1984, Baisan y Swetnam 1990, Brown et al. 1999, Brown y Sieg 1996, 1999).

En este trabajo las cicatrices de fuego fueron definidas como heridas en la base de los troncos, identificadas por la presencia de carbón y por la respuesta del crecimiento del leño a la muerte de una sección del cambium vascular (Sutherland 1997, Baker 1989, Kitzberger et al. 2000).

Análisis de los regímenes de fuego. Todas las cicatrices fueron fechadas y compiladas en una cronología compuesta, a la cual se le calcularon los intervalos medios entre fuegos para el período completo de la cronología y para distintos períodos históricos.

Se calcularon los intervalos medios entre fuegos (IMF) y los intervalos con probabilidad mediana de Weibull (IPMW). El IMF es el número promedio de años entre fechas de fuego en una cronología compuesta y ha sido ampliamente usado para describir frecuencias de fuego. La varianza en los intervalos de fuego está representada por la primera desviación estándar y por la serie de intervalos. Por ello, las conclusiones estadísticas derivadas a partir del uso del IMF requieren una distribución normal de los intervalos entre fuegos. En general, los datos de intervalos entre fuegos son sesgados positivamente debido a que no existe un límite superior de intervalo, mientras que un año es el intervalo inferior posible entre fuegos (Johnson y Wagner 1985).

El IPMW es el intervalo entre fuegos asociado con el $50 \%$ de la probabilidad de una distribución de tipo Weibull de todos los intervalos entre fuego de una cronología, en la cual la varianza está representada por los intervalos que exceden las probabilidades del $5 \%$ y el $95 \%$, y es considerada como una estimación menos sesgada de la tendencia central en las distribuciones de intervalos entre fuegos (Grissino-Mayer 1999, Brown y Sieg 1999). El IMF 
y el IPMW alcanzan el mismo valor si los intervalos entre fuegos presentan una distribución normal.

Se trabajó con el programa FHX2 (Grissino-Mayer 1995) para calcular los valores del IMF y del IPMW. Éstos fueron calculados analizando la totalidad de los fuegos ocurridos en el área de estudio y a partir del análisis de los fuegos mayores. Los fuegos mayores fueron definidos como aquellos que fueron registrados por al menos el $20 \%$ de los caldenes analizados (Kitzberger et al. 2000). Los fuegos probablemente fueron más extensos espacialmente durante los fuegos mayores que durante fuegos que fueron registrados por sólo unos pocos árboles (Guyette y Cutter 1991, Sutherland 1997).

Los IMF y los IPMW fueron calculados para el período de ocupación aborigen (antes de 1879) y para el período a partir de la colonización. Este último fue dividido para el análisis en primer período (1880-1930) y último período (1930-1989) en base a una fuerte crisis ambiental ocurrida durante las décadas 1930-1940 en la región (Viglizzo y Roberto 1991).

La estacionalidad asignada a las cicatrices de fuego fueron: fuegos de primavera (cicatrices ubicadas dentro de la banda de leño temprano), fuegos de verano (cicatrices ubicadas dentro de la banda de leño tardío) y fuegos de otoño-invierno (cicatrices ubicadas en el límite entre dos anillos) (Dieterich y Swetnam 1984, Baisan y Swetnam 1990). En base a los datos fenológicos de esta especie (Cano et al. 1985), las cicatrices de fuego situadas en el leño temprano habrían sido originadas por fuegos ocurridos entre los meses de octubre y noviembre, mientras que las ubicadas en el leño tardío corresponderían a fuegos ocurridos entre diciembre y abril. Las ubicadas en el límite entre dos anillos habrían sido originadas por fuegos ocurridos entre mayo y septiembre.

\section{RESULTADOS}

Treinta y una de las treinta y cinco muestras trabajadas pudieron ser correctamente cofechadas, obteniéndose una cronología de ancho de anillos desde 1788 a 1995 para el sitio Bajo Verde. Se reconocieron 164 cicatrices de fuego, las que representaron un total de 50 eventos de fuego en la cronología. La primera cicatriz de fuego registrada en la cronología fue en el año 1795 y la última en 1989, habiéndose originado una cronología de fuegos que cubre parte del período de ocupación aborigen (antes de 1879) y el período a partir de la colonización y hasta 1989 (figura 1).

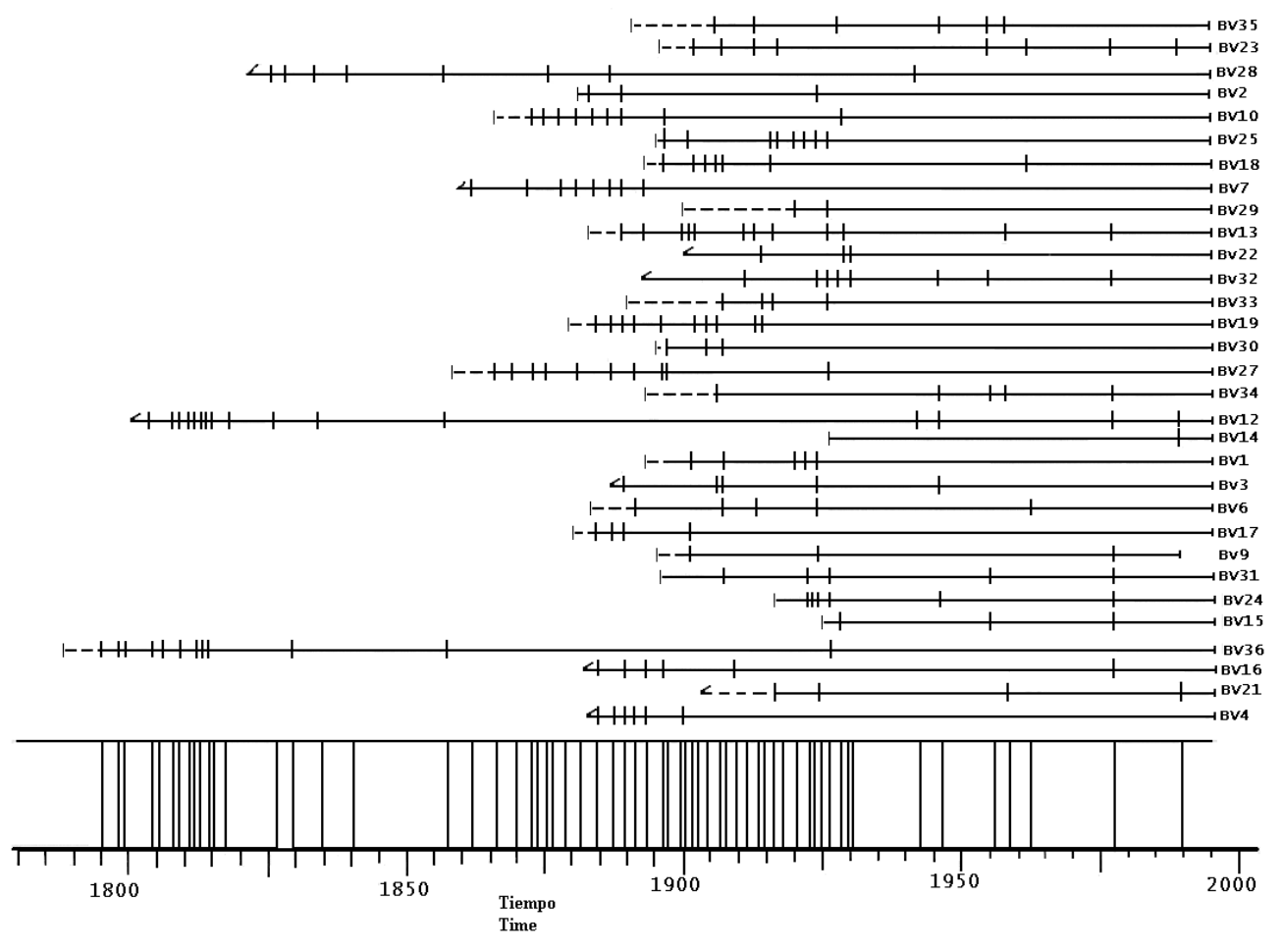

Figura 1. Cronología de fuego del Bajo Verde, provincia de La Pampa, Argentina. Las líneas horizontales representan el tiempo de vida de cada uno de los caldenes donde se representa el año de nacimiento y muerte del ejemplar (línea vertical al final de la serie) y las cicatrices de fuego (líneas verticales). Las líneas punteadas indican el periodo de vida anterior a la existencia de cicatrices de fuego en el leño. Debajo, cronología compuesta en la cual las líneas verticales indican la ocurrencia de cicatrices de fuego en al menos una muestra en el área de estudio.

Fire chronology of Bajo Verde, La Pampa province, Argentina. Horizontal lines correspond to the life time of each sample from their first year until death year (indicated by short vertical bars at the end of the series) and fire scars (vertical bars). Dashed lines indicate the life time years prior to appearance of fire scars in wood. Below, the composite fire chronology of the area, where vertical bars indicate fire occurrence in at least one sample. 
El IMF para el área de estudio desde el primer fuego registrado en 1795 hasta el último en 1989 fue de 3,96 años (con intervalos mínimo y máximo entre fuegos de 1 y 17 años respectivamente). Los intervalos entre fuegos se ajustaron más adecuadamente a una distribución de tipo Weibull. El IPMW para el mismo período fue de 3,21 años, con intervalos significativos menores y mayores entre fuegos de 0,64 y 7,93 años respectivamente.

$\mathrm{El}$ análisis de la frecuencia de los fuegos mayores o más extensos (fuegos en los que al menos el $20 \%$ de los caldenes registraron el evento) arrojó un valor del IPMW de 4,97 años, demostrando que los mismos fueron muy frecuentes en el área de estudio (cuadros 1 y 2).

Cuadro 1. Valores de la frecuencia de fuego de Bajo Verde para los distintos períodos analizados.

Values of fire frequency for Bajo Verde site at different periods.

\begin{tabular}{rccccc}
\hline Período & $\begin{array}{c}\mathrm{N}^{\circ} \text { de } \\
\text { intervalos }\end{array}$ & $\begin{array}{c}\mathrm{IMF} \pm \mathrm{DE} \\
(\mathrm{años})^{5}\end{array}$ & $\begin{array}{c}\text { Rango de } \\
\text { intervalos } \\
\text { (años) }\end{array}$ & $\begin{array}{c}\text { IPMW } \\
{\text { (años })^{6}}^{6}\end{array}$ & $\begin{array}{c}\text { Varianza de } \\
\text { Weibull (años) }\end{array}$ \\
\hline $1795-1989^{1}$ & 49 & $3,6 \pm 3,84$ & $1-17$ & 3,21 & $0,4-7,93$ \\
$1795-1879^{2}$ & 16 & $5,18 \pm 4,37$ & $1-16$ & 4,40 & $0,68-11$ \\
$1880-1930^{3}$ & 25 & $1,96 \pm 0,89$ & $1-4$ & 1,92 & $0,76-3,22$ \\
$1930-1989^{4}$ & 7 & $8,42 \pm 4,79$ & $3-15$ & 7,13 & $2,10-16,7$ \\
\hline
\end{tabular}

${ }^{1}$ Período completo de la cronología de fuegos. 2Período de ocupación aborigen. ${ }^{3}$ Primer período a partir de la colonización. ${ }^{4}$ Último período a partir de la colonización. ${ }^{5}$ Intervalo medio entre fuegos y desviación estándar. ${ }^{6}$ Intervalo con la probabilidad mediana de Weibull $(50 \%)$. ${ }^{7}$ Intervalos con el 5\% y $95 \%$ de la probabilidad de Weibull.

Cuadro 2. Valores de la frecuencia de fuego en Bajo Verde calculados a partir de los fuegos mayores (fuegos registrados por al menos el $20 \%$ de los caldenes analizados).

Values of frequency of major fires for Bajo Verde site at different periods.

\begin{tabular}{rccccc}
\hline Período & $\begin{array}{c}\mathrm{N}^{\circ} \text { de } \\
\text { intervalos } \\
\text { (años) }\end{array}$ & $\begin{array}{c}\text { IMF } \pm \text { DE } \\
(\text { años })^{5}\end{array}$ & $\begin{array}{c}\text { Rango de } \\
\text { intervalos } \\
\text { (años) }\end{array}$ & $\begin{array}{c}\text { IPMW } \\
(\text { años })^{6}\end{array}$ & $\begin{array}{c}\text { Varianza de } \\
\text { Weibull (años) }\end{array}$ \\
\hline $1795-1989^{1}$ & 27 & $6,74 \pm 9,96$ & $1-51$ & 4,97 & $0,91-12,91$ \\
$1795-1879^{2}$ & 15 & $6,92 \pm 8,85$ & $1-17$ & 4,88 & $1,13-11,12$ \\
$1880-1930^{3}$ & 10 & $5,53 \pm 4,32$ & $2-14$ & 2,78 & $0,79-5,65$ \\
& & & - & - & - \\
$1930-1989^{4}$ & $\begin{array}{c}\text { Solo fuego } \\
1977\end{array}$ & - & - & & -
\end{tabular}

${ }^{1}$ Período completo de la cronología de fuegos. ${ }^{2}$ Período de ocupación aborigen. ${ }^{3}$ Primer período a partir de la colonización. ${ }^{4}$ Último período a partir de la colonización. ${ }^{5}$ Intervalo medio entre fuegos y desviación estándar. ${ }^{6}$ Intervalo con la probabilidad mediana de Weibull (50\%). ${ }^{7}$ Intervalos con el 5\% y $95 \%$ de la probabilidad de Weibull.
La frecuencia de fuego en el área de estudio ha sido muy dinámica en el tiempo y ha experimentado cambios importantes probablemente en relación a los cambios en la ocupación y en el uso de estas tierras boscosas (figura 2).

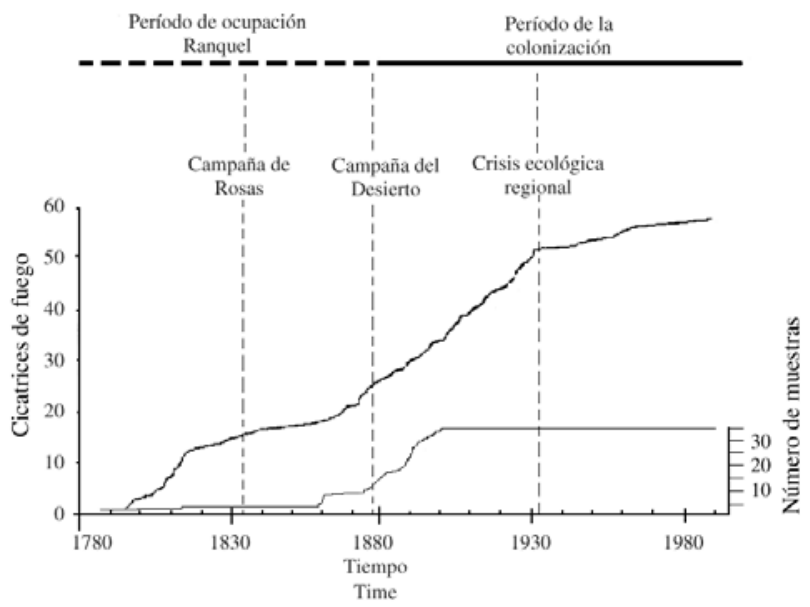

Figura 2. Distribución acumulada de ocurrencia de fuegos en bosque de caldén del Bajo Verde, provincia de La Pampa, Argentina.

Accumulated distribution of fire occurrence in a caldén woodland in Bajo Verde, La Pampa province, Argentina.

El $81 \%$ de las cicatrices de fuego se ubicaron dentro de la banda del leño tardío de los anillos de crecimiento, mientras que un $18 \%$ de ellas estaban situadas en la banda de leño temprano de los mismos. Sólo al $1 \%$ de las cicatrices de fuego no fue posible asignar ubicación dentro del anillo de crecimiento por causas de erosión y/o daño de la madera.

\section{DISCUSIÓN}

La determinación de las frecuencias de fuego en los diferentes períodos analizados muestra una alta variabilidad de este disturbio en el tiempo. Para el período de ocupación Ranquel (antes de 1879), el valor del IPMW fue de 3,12 años, con intervalos significativos menores y mayores entre fuegos de 0,38 y 11 años respectivamente. En un análisis más detallado de este período se pueden identificar distintos regímenes de fuegos; durante los primeros años de la cronología y hasta 1840, la alta presencia de cicatrices de fuego estaría demostrando la existencia de un régimen de fuegos de alta frecuencia. A pesar de que sólo tres caldenes cubren este período de tiempo, estaban distanciados entre ellos en el área de estudio en aproximadamente $1 \mathrm{~km}$. Estos bosques estaban habitados por aborígenes Ranqueles desde mediados del siglo XVIII. De las Casas (1910) describe que Calchague, un sitio 
ubicado a $5 \mathrm{~km}$ del sitio de muestreo, estaba ocupado por 10 aborígenes y sus familias en el año 1779, y De la Cruz (1969) relata que para 1806 los alrededores de Calchague estaban altamente poblados por Ranqueles. No se conoce información acerca del uso del fuego en estas etapas por parte de los Ranqueles más que para producir señales de humo para su comunicación (Cazenave 1995), y para la caza del guanaco (Lama guanicoe Mull.) y otra fauna menor (Cox 1863 y Guinnard 1941).

Durante las décadas de 1840-1850 se observa un cambio en el régimen de fuegos en el área de estudio. Los fuegos fueron menos frecuentes y, como lo reflejan las muestras BV12, BV36 y BV28 (figura 1), de gran extensión en el área de estudio. Es altamente probable que Calchague y sus alrededores hayan sido abandonados temporalmente por los Ranqueles durante estas décadas, a causa de campañas militares realizadas en esta zona durante los años 1833-34-35, las que redujeron las poblaciones Ranqueles e indujeron la dispersión y el reacomodamiento de los sobrevivientes (Walther 1976).

A partir de 1860 y hasta 1879 , año en que los Ranqueles culminan definitivamente su ocupación en los bosques de caldén a causa de la "conquista del desierto", se observa una alta frecuencia de fuegos. El uso del fuego por parte de los Ranqueles y los soldados ha sido registrado históricamente como estrategia de defensa durante esta última etapa de ocupación aborigen (Ambrosetti 1893, Racedo 1965, Mansilla 1966 y Ebelot 1968). En la figura 2 se pueden observar estos cambios en la frecuencia de fuegos, representados por la variación en la pendiente de la curva, durante el período de ocupación Ranquel.

Para el período de la colonización se observan dos regímenes de fuego marcadamente diferentes en el área de estudio. El primero, a partir de la colonización de la zona (1880-1930), presenta un régimen de fuegos de alta frecuencia (IPMW de aproximadamente dos años, con un intervalo máximo significativo entre fuegos próximo a los tres años) y una frecuencia de fuegos extensos o mayores de aproximadamente tres años, con un intervalo máximo significativo próximo a los seis años.

El fuego fue ampliamente utilizado durante el primer período a partir de la colonización de la zona. No existen crónicas históricas que documenten este tipo de prácticas y sus objetivos. Sin embargo, es factible pensar que el fuego era usado como una herramienta auxiliar para la conversión de tierras boscosas en tierras agrícolas, para controlar la invasión de leñosas y arbustos en las tierras agrícolas y para incrementar la disponibilidad y la calidad del forraje para el pastoreo del ganado (González et al. 2005).

Durante las décadas de 1930-40 ocurrieron en la región fuertes adversidades climáticas, las que causaron importantes pérdidas de suelos, cultivos y ganados y una gran emigración poblacional. Todos estos factores, acompañados del sistema de explotación no conservacionista de la agricultura y la ganadería, resultaron en una fuerte crisis regional que alcanzó su momento culminante a mediados de la década de 1930 y que marcó un cambio en la tendencia de la calidad del ecosistema de la región a partir de una concientización del sector rural y de instituciones asociadas (Viglizzo y Roberto 1991).

El segundo, durante el último período analizado (19301989), presenta el IPMW de mayor valor (siete años), con el más largo intervalo máximo significativo entre fuegos (aproximadamente 17 años) de la cronología de fuegos para el área de estudio. El único fuego registrado por al menos el $20 \%$ de los caldenes analizados fue el del año 1977, lo que demuestra que los fuegos fueron de poca extensión en el área de estudio durante este período, probablemente a causa de la pronta supresión del fuego por parte del hombre, resultando en una minimización del tamaño de éste.

El régimen de fuegos de este período en el área de estudio se caracterizó por fuegos menos frecuentes y de menor extensión. En la figura 2 se observa la variación de la pendiente de la línea de fuegos acumulados a partir de 1930, reflejando el cambio de la frecuencia de los fuegos. La baja frecuencia de fuegos durante las últimas décadas está probablemente relacionada con el gran esfuerzo realizado por instituciones gubernamentales (Dirección de Defensa Civil y Dirección de Bosques del Gobierno de la Provincia de La Pampa y Plan Nacional de Manejo del Fuego) para la supresión del fuego en estos bosques y el control que ellas ejercen sobre las prácticas de quemas y retaceos en los campos.

La ubicación predominante de las cicatrices de fuego en las bandas del leño temprano (18\%) y del leño tardío $(81 \%)$ de los anillos de crecimiento demuestra una marcada estacionalidad (primavera-verano) del fuego. Estos meses (noviembre a marzo) se caracterizan por la presencia de altas temperaturas, fuertes vientos, baja humedad y gran cantidad de material vegetal combustible acumulado en los estratos inferiores del bosque. El desarrollo de tormentas eléctricas convectivas es frecuente durante estos meses y los rayos son comunes. Estos pueden originar igniciones aisladas y de poca extensión o incendios muy extensos, como los del verano de los años 2000-2001, durante los cuales se quemaron en la provincia de La Pampa más de 3.500 .000 ha. Por otro lado, los productores vienen haciendo uso del fuego durante estos meses para la conversión de tierras boscosas en tierras agrícolas y para el control de la invasión de especies leñosas. El caldén resulta más susceptible al fuego durante su período de crecimiento (noviembre a abril) que en los meses en los que se encuentra en reposo (abril a octubre) (Llorens 2000).

Los fuegos de primavera y verano son utilizados en la zona para inducir el rebrote tierno de pastos duros (Stipa tenuisima Trinius, S. ginerioides Philippi) y para incrementar la cobertura de especies forrajeras (Stipa tenuis Philippi, S. longiglumis Philippi, Piptochaethium napostaense (Speg.) Hackel, Poa ligularis Poiret) con objetivos ganadero-pastoriles. 
A causa de que el $44 \%$ de los fuegos en la provincia de La Pampa son originados por tormentas eléctricas (Scarone 1990, Irigoin et al. 1996), otro de los objetivos con los que los productores vienen haciendo uso del fuego en sus campos es reducir la probabilidad de ocurrencia de fuegos catastróficos y de difícil control.

Los resultados alcanzados en este trabajo corroboran lo expuesto por Lell (1990), Scarone (1994), Steibel y Troiani (1999) y Llorens (2000), quienes sostienen la existencia de una sistemática recurrencia del fuego durante la colonización de la región. Estos autores atribuyen a esta recurrencia del fuego la fachinalización del histórico bosque abierto de caldén.

Por otro lado, Cano et al. (1985), Anchorena (1998), Boó (1990), Gorondi (1990), Sipowicz (1994) y Buzzo (1997) sostienen la existencia de una fuerte reducción de la frecuencia de fuegos a partir de la colonización europea. Según los autores, la supresión del fuego en el bosque habría promovido los cambios estructurales sufridos por los bosques de caldén. Es muy probable que estos autores estén haciendo referencia a la reducción de la frecuencia del fuego sufrida en la región a partir de la década de 1930.

Interpretar los bosques actuales o evaluar su restauración o la planificación de su manejo racional implica reconocer su naturaleza dinámica. Las perspectivas históricas incrementan nuestro entendimiento de la naturaleza dinámica de los paisajes, la influencia a largo plazo del ser humano como componente de los ecosistemas funcionales y la base cultural de decisiones de conservación.

\section{CONCLUSIONES}

El análisis dendrocronológico de cicatrices de fuego registradas en los leños del caldén brinda valiosa información histórica de diversas variables de los regímenes de fuego que afectan a estos bosques, entre ellas la frecuencia, estacionalidad y extensión.

El régimen de fuego ha sido altamente dinámico durante los siglos XIX y XX en el área de estudio. Tanto la frecuencia como la extensión de los fuegos han experimentado importantes cambios, probablemente en relación al tipo de ocupación y del uso del área.

La estacionalidad de los fuegos, principalmente de verano y primavera, ha sido la única característica del régimen de este disturbio que se ha mantenido constante durante los últimos 200 años.

El bosque actual de caldén es el reflejo de un régimen de fuego dinámico y lleva en él la memoria de diferentes regímenes de este disturbio.

$\mathrm{Si}$ bien frecuentemente se utiliza el período preeuropeo o precolonización como marco de referencia para la comparación con las condiciones actuales, en este trabajo se pudo comprobar que no hubo un único régimen de fuegos en los bosques de caldén durante el período de precolonización o período de ocupación Ranquel, aunque éste parece no haber sufrido cambios tan marcados como en el pasado más reciente.

\section{REFERENCIAS}

Ambrosetti JB. 1893. Viaje a la Pampa Central. Viedma, Argentina. Imprenta Martín. p. 16-44.

Anchorena JBN. 1998. Manejo en regiones semiáridas. Pastizales Naturales de La Pampa, Tomo II. CREA. Convenio AACREAProvincia de La Pampa, Argentina. 112 p.

Baisan CH, TW Swetnam. 1990. Fire history on a desert mountain range: Rincon Mountain Wilderness, Arizona, USA. Canadian Journal of Forest Research 20: 1559-1569.

Baker WL. 1989. Effects of scale and spatial heterogeneity on fire-interval distributions. Can. J. For. Res. 19: 700-706.

Boó RM. 1990. Algunos aspectos a considerar en el empleo del fuego. Revista de la Facultad de Agronomía (La Pampa, Argentina) 5: 63-80.

Boó RM, DV Peláez, SC Bunting, MD Mayor, OR Elía. 1997. Effect of fire on woody species in central semi-arid Argentina. Journal of Arid Environments 35: 87-94.

Brown PM, CH Sieg. 1996. Fire history in interior ponderosa pine communities of the Black Hills, South Dakota, USA. Journal of Wildland Fire 6: 97-105.

Brown PM, CH Sieg. 1999. Historical variability in fire at the ponderosa pine-Northern Great Plains prairie ecotone, southeastern Black Hills, South Dakota. Ecoscience 6(4): 539-547.

Brown PM, TW Swetnam. 1993. A cross-dated fire history from coast redwood national park, California. Can. J. For. Res. 24: 21-31.

Brown PM, MR Kaufmann, WD Shepperd. 1999. Long-term, landscape patterns of past fire events in a montane ponderosa pine forest of central Colorado. Landscape Ecology 14: $513-532$.

Buzzo CA. 1997. Toward an increased and sustainable production in semi-arid rangeland of central Argentina: 2 decades of research. Journal of Arid Environment 36: 197-210.

Cabrera AL, A Willink. 1973. Biogeografía de América Latina. Serie Biología. Monogr.13. Organization of American States, Washington DC. $122 \mathrm{p}$.

Cano E, HD Estelrich, H Holgado. 1985. Acción del fuego en los estratos graminosos y arbustivos de un bosque de caldén. Revista de la Facultad de Agronomía (La Pampa, Argentina) 1: 81-96.

De las Casas D. 1910. Noticia individual de los caciques y capitanes pegüenches y pampas que residen al sud (...). In De Anguelis P ed. Colección de obras y documentos. $2^{\mathrm{a}}$ ed. Buenos Aires, Argentina. Librería Nacional, J Lajouane, Parte 4. p. 584-587.

Cazenave W. 1995. Señales de fuego y humo en Pampa y Patagonia. Periódico La Arena, suplemento cultural "Caldenia", Santa Rosa, La Pampa, Argentina.

Cox G. 1863. Viaje en las regiones septentrionales de la Patagonia. 1862-1863. Anales de la Universidad de Chile 23: 3-239.

De la Cruz L. 1969. Viaje a su costa del alcalde provincial del muy ilustre Cabildo de La Concepción de Chile, ...Incluye Diario de viaje, descripción de la naturaleza de los te- 
rrenos, (...). In De Anguelis P ed. Colección de obras y documentos. Buenos Aires, Argentina. Editorial Plus Ultra. Parte 2. p. 7-491.

Dieterich JH, TW Swetnam. 1984. Dendrochronology of a firescarred Ponderosa Pine. Forest Science, 30: 238-247.

Ebelot A. 1968. Recuerdos y relatos de la Guerra de Fronteras. Buenos Aires, Argentina. Editorial Plus Ultra. 240 p.

González M, T Veblen, J Sibold. 2005. Fire history of AraucariaNothofagus forests in Villarrica National Park, Chile. Journal of Biogeography 32: 1187-1202.

Gorondi AZ. 1990. La reintroducción del fuego en el ecosistema bosque de caldén. Revista de la Facultad de Agronomía (La Pampa, Argentina) 5: 149-154.

Grissino-Mayer HD. 1995. Tree-ring reconstructions of climate and fire history at El Malpais National Monument, New Mexico. Ph.D. dissertation. Tucson, Arizona. The University of Arizona. 407 p.

Grissino-Mayer HD. 1999. Modeling fire interval data from the American Southwest with the Weibull distribution. International Journal of Wildland Fire 9: 37-50.

Guinnard A. 1941. Tres años de esclavitud entre los patagones. Relatos de mi cautiverio. Buenos Aires, Argentina. Espasa Calpe. 169 p.

Guyette RP, BE Cutter. 1991. Tree ring analysis of fire history of a post oak savanna in the Missouri Ozarks. Natural Areas Journal 11: 93-99.

Head FB. 1918. Las Pampas y los Andes. Notas de Viaje. Buenos Aires, Argentina. Biblioteca de la Nación. p. 30-35.

Holmes RL. 1983. Computer-assisted quality control in tree-ring dating and measurement. Tree-Ring Bulletin 43: 69-75.

Irigoin N, H Zucchini, R Panchuk, M Scarone. 1996. Evaluación de pérdidas económicas y uso del fuego por productores agropecuarios de La Pampa. Dirección de Producción Forestal, SAG y Direcciones de Recursos Naturales y de Producción Forestal del Ministerio de la Producción de La Pampa, Argentina. 18 p.

Johnson EA, CE Wagner van. 1985. The theory and use of two fire history models. Can. J. For. Res. 15: 214-220.

Kitzberger T, TT Veblen, R Villalba. 2000. Métodos dendrocronológicos y sus aplicaciones en estudios de dinámica de bosques templados de Sudamérica. Dendrocronología en América Latina. F. A. Roig (compilador), EDIUNC, Mendoza, Argentina. pp. 17-78.

Lara A, S Fraver, J Aravena, A Wolodarsky-Franke. 1999. Fire and the dynamics of Fitzroya cupressoides (alerce) forests of Chile's Cordillera Pelada. Ecoscience 6: 100-109.

Lara A, A Wolodarsky-Franke, J Aravena, M Cortes, S Fraver, F Silla. 2003. Fire regimes and forest dynamics in the Lake Region of south-central Chile. In Veblen T, W Baker, G Montenegro, T Swetnam eds. Fire and climatic change in temperate ecosystems of the western Americas. New York, USA. Springer. Ecological Studies 160: 322-342.

Lehtonen H, P Huttunen, P Zetterberg. 1996. Influence of man on forest fire frequency in North Karelia, Finland, as evidenced by fire scars on Scots pines. Annales Botanici Fennici 33: 257-263.

Lell JD. 1990. Incendios de bosques en La Pampa. Revista de la Facultad de Agronomía (La Pampa, Argentina) 5(1): 29-33.
Llorens EM. 2000. Estrategias de manejo del caldenal para lograr una máxima producción sustentable. Nuestro Campo. p. 13-17.

Lorimer CG. 1985. Methodological considerations in the analysis of forest disturbance history. Can. J. For. Res. 15(1): 200-213, 1985.

Mansilla L. 1966. Expedición a los Indios Ranqueles. Buenos Aires, Argentina. Kapelusz. p. 134-135.

McBride JR. 1983. Analysis of tree rings and fire scars to establish fire history. Tree-Ring Bulletin 43: 51-67.

Medina AA, EG Dussart, HD Estelrich, EA Morici. 2000. Reconstrucción de la frecuencia de fuego en un bosque de Prosopis caldenia Burkart, de Arizona, sur de la Provincia de San Luis. Multequina 9: 91-98.

Medina AA. 2003. Reconstrucción de historias de fuego en bosques mediante técnicas dendrocronológicas. In Kunst CR, S Bravo, JL Panigatti eds. Fuego en los Ecosistemas Argentinos. Santiago del Estero, Argentina. INTA. p. 133-143.

Monticelli JV. 1983. Anotaciones fitogeográficas de La Pampa Central. Lilloa, III. Argentina. 382 p.

Racedo E. 1965. Memoria Militar y descriptiva de la $3^{a}$ división expedicionaria. Buenos Aires, Argentina. Editorial Plus Ultra. $231 \mathrm{p}$.

Scarone ML. 1990. Consideraciones sobre el fuego como herramienta de manejo en el bosque de caldén. Revista de la Facultad de Agronomía (La Pampa, Argentina) 5(1): 161-167.

Scarone M. 1994. Incendios Forestales. Agro Pampeano 27:5-15.

Schulman E. 1956. Dendroclimatic change in semiarid America. Tucson, Arizona. University of Arizona Press. 142 p.

Sipowicz AH. 1994. Ecología y manejo del fuego en el ecosistema del caldenal. Estación Experimental Anguil- INTA-Anguil, La Pampa, Argentina. Boletín de divulgación técnica $N^{\circ} 51$. p. 16.

Steibel PE, OT Troiani. 1999. El género Prosopis (Leguminosae) en la Provincia de La Pampa. Revista de la Facultad de Agronomía (La Pampa, Argentina) 10 (2): 24-48.

Sutherland EK. 1997. History of fire in a Southern Ohio SecondGrowth Mixed-Oak Forest. Proceedings of the 11 Central Hardwood Forest Conference, March 23-26, Columbia, Missouri. pp. 172-183.

Viglizzo EF, ZE Roberto. 1991. Evolución y tendencia del agroecosistema en la pampa semiárida. In Seminario Juicio a nuestra agricultura. Hacia el desarrollo de una agricultura sostenible. Buenos Aires, Argentina. INTA. Editorial Hemisferio Sur. p. 85-99.

Walther JC. 1976. La Conquista del Desierto. Lucha de frontera contra el indio. Buenos Aires, Argentina. Eudeba. 629 p.

Whelan R J. 1995. The Ecology of Fire. Cambridge University Press. 346 p.

Willard R, CM McKell. 1977. Efecto de la quema controlada en árboles y arbustos de monte. Empleo del Fuego para la Conversión del Monte, en la Región Semiárida Pampeana. Universidad Nacional del Sur. Departamento de Ciencias Agrarias. CAFPTA. Argentina. p. 34-37. 